\title{
Topology Based Flow Analysis and Superposition Effects
}

\author{
Julia Ebling ${ }^{1}$, Alexander Wiebel ${ }^{2}$, Christoph Garth ${ }^{3}$, and Gerik \\ Scheuermann ${ }^{4}$ \\ 1 Universität Leipzig ebling@informatik.uni-leipzig.de \\ 2 Universität Leipzig wiebel@informatik.uni-leipzig.de \\ 3 TU Kaiserslautern garth@informatik.uni-kl.de \\ 4 Universität Leipzig scheuer@informatik.uni-leipzig.de
}

Summary. Using topology for feature analysis in flow fields faces several problems. First of all, not all features can be detected using topology based methods. Second, while in flow feature analysis the user is interested in a quantification of feature parameters like position, size, shape, radial velocity and other parameters of feature models, many of these parameters can not be determined using topology based methods alone. Additionally, in some applications it is advantageous to regard the vector field as a superposition of several, possibly simple, features. As topology based methods are quite sensitive to superposition effects, their precision and usability is limited in these cases. In this paper, topology based analysis and visualization of flow fields is estimated and compared to other feature based approaches demonstrating these problems.

\section{Introduction}

Visualization and analysis of vector fields from flow simulations and measurements is an important step in engineering processes, e.g. during the design phase of airplanes, cars, trains, and combustion chambers. According to Dr. Shneiderman's Visual Information Seeking Mantra [13] visualization of data consists of three steps: "Overview first, zoom and filter, then details-ondemand". However, due to the extreme size and complexity of today's data sets it may not be possible to provide an overview of the data. Interactive zooming and filtering also quickly reaches its limits. Thus the pipeline has to be extended by an automatic analysis step to reduce size and/or complexity of the data before trying to visualize it. This results in Keim's Visual Analytics Mantra "Analyse first, show the important, zoom, filter and analyse further, details on demand" that he proposed at the Workshop on Visual Analytics 2005 in Darmstadt.

Up to now topology has mainly been used to give an overview of a flow as it segments a data set into regions of same flow behavior. In this paper, 


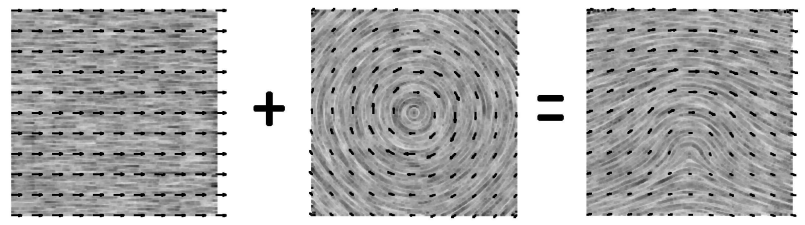

Fig. 1. Linearity of features - the superposition principle: The flow on the right is the direct sum of the two different flows on the left.

advantages as well as deficits of topological methods concerning the analysis step mentioned in the previous paragraph are discussed. Therefore, topology is compared to feature based methods. We do not only treat topology as a description of the flow, but also analyze topology within the context of typical feature models like the Vatistas vortex [17]. Concerning this model the ability of topology based methods to determine parameters of feature models is analyzed (Section 3).

For signal processing, linear, shift invariant signals play an important role as most signals can be described, or at least approximated, by them. Other signals can be approximated quite well, too. The linearity property is also known as the superposition principle, stating that a combination of signals is equal to the same combination applied to all parts of these signals. This means that a complex signal can be understood as a linear combination of several simpler signals (Figure 1). Keeping this signal processing perspective in mind, we can treat vector fields as superpositions too and thus can describe phenomena like vortices hidden in a strong homogeneous flow. As topology is based on critical points, that is points where the velocity in the field is zero, it is quite sensitive to changes of the mean flow of a data set. Adding or subtracting, i.e. superposing, different constant flows to a flow, thus will change its topology. One way to avoid these changes is to analyze the localized or region-specific flow [18] which is independent of constant and homogenous flows passing through the considered region. Superposition effects, however, can also appear independent of such flow components, e.g. when two vortices overlap. In Section 4 we discuss superposition phenomena and their influence on topology based methods and compare the superposition view to the usual perception of vector fields describing the actual flow.

\section{Feature Definitions}

Features are often defined as "phenomena, structures or objects in a data set, that are of interest for a certain research or engineering problem" [9]. It is not possible to give a list of all features of interest for flow fields in general as these differ from application to application and small changes of one feature can lead to a variety of new features. Nevertheless, most features can be categorized into a few groups like vortices and other swirling flows, shock waves, shear flow 
and boundary layers, reversed flow, saddle points, separation and attachment lines or surfaces, areas with convergent or divergent behavior, and regions with homogeneous or constant flow. The remainder of this section gives a short overview of feature definitions we will use in the following discussion.

\subsection{Vector Field Topology}

In a vector field, as already mentioned, positions where the magnitude of the vectors is zero are called critical points. Critical points can be classified by the eigenvalues and eigenvectors of the velocity gradient tensor at their position. In linear 2D vector fields, they are classified as sinks, sources, saddles and center points. Sinks and sources can be of the types focus, spiral, node or improper node. Critical points are connected by streamlines called separatrices which divide the vector field into regions of same flow behavior. The topology graph of a vector field consists of all critical points, separatrices and closed streamlines of the vector field.

\subsection{Correlation Methods}

Vortices, shear flow, sources, sinks, saddles, separation and attachment lines, and homogeneous flow can all be coded into vector valued masks (for examples see Figure 2). Using these masks for (rotation invariant) pattern matching based on correlation allows to detect similar areas in a vector field $[2,5]$. A disadvantage of this approach is the computational time for irregular grids. For regular grids, acceleration via fast Fourier transform can be used [3]. The main advantage of this approach is the robustness to noise due to the averaging in the correlation. This is of great importance for measured data. Furthermore, as smoothing can be described by a convolution with a scalar valued mask, it is commutative with the pattern matching. When the average of a mask is zero, the matching results are independent of any mean flow of the vector field. As the masks are linear, shift invariant filters they are suitable for the analysis of superposition phenomena. Feature definitions using vorticity can be integrated into this approach as derivation and derived quantities can be computed via convolution with a mask $[2,3]$.

\subsection{Threshold Based Feature Definitions}

Thresholds for high vorticity (curl of velocity, $\nabla \times v$ ), high helicity (projection of vorticity onto velocity, $(\nabla \times v) \cdot v)$ and low modified pressure (negative $\lambda_{2}$, see [6]) are often used to determine position, size and shape of vortices. In addition to these, there is a variety of vortex core detection algorithms of all kinds. An overview can be found in $[9,11]$. Vorticity and $\lambda_{2}$ are Galilean invariant, that is, they are independent of the frame of reference and thus independent of any mean flow. Computing vorticity is also commutative with smoothing, that is, computing vorticity from smoothed data yields the same results as computing it first and performing smoothing afterwards. 

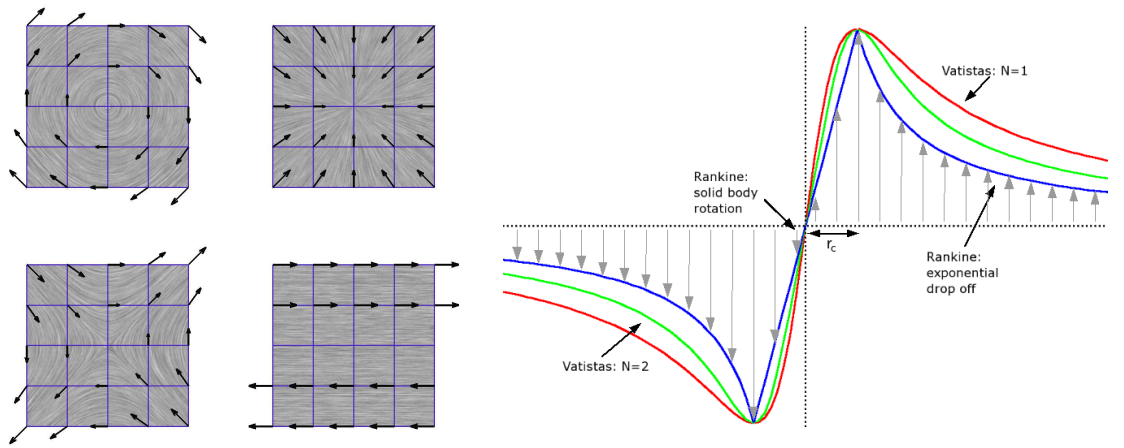

Fig. 2. Left: Rotation, convergence, saddle and shear, each visualized using hedgehogs and LIC. Right: The Vatistas vortex model. A pure, circular rotation is assumed. A cut through this rotation results in the described velocity profile.

\section{Quantification of Feature Parameters}

For detailed flow visualization, a preceding analysis of the flow and its features is often necessary. In this section, the advantages and limits of topological methods for an analysis of features are discussed and compared to those of other feature based approaches. All properties are demonstrated using the Vatistas vortex [17] (Figure 2), a popular vortex model in fluid dynamics, as an example.

\subsection{Feature Models - an Example}

The definition of a Vatistas vortex [17] is based on a pure, circular rotation with the magnitude of the vectors given by

$$
v(r)=2^{1 / N} r_{c}^{2} \frac{v_{c} r}{\left(r_{c}^{2 N}+r^{2 N}\right)^{1 / N}},
$$

where $r_{c}$ is the vortex core radius, $r$ the distance to the vortex core, $v_{c}$ the velocity at the vortex core radius, and $N$ a parameter describing the transition from the linear velocity profile within the vortex core to the exponential drop-off of the velocity magnitudes outside. For $N=1$, the Vatistas model equals the Scully vortex model [12] and for $N=\infty$, the Rankine model [10] is obtained. Important parameters which engineers want to determine in this model are vortex core center or vortex position, vortex core radius, the circumferential velocity at the vortex core radius, the circumferential velocity distribution, overall vorticity within the vortex core and, in $3 \mathrm{D}$, the maximal axial velocity and the axial velocity distribution $[1,16]$.

For the Vatistas vortex, the vectors describing the flow are more important than the actual streamlines. The velocity of the flow is not only indispensable for the engineers whereas it is neglected in topological methods, but the 
projection of the vectors describing the actual flow onto the vectors describing the Vatistas vortex can be more important than the flow itself. Due to this projection, the topology of this vortex can differ greatly according to the remaining part of the flow (Section 4).

The Vatistas vortex is an abstraction of vortices. Though tunable by some parameters like vortex core radius and velocity distribution, this model assumes a perfectly circular vortex and thus only approximates real flow (Figure 2). The vortex is assumed to spread out infinitely, though the influence of the vortex will converge to zero with increasing distance to the center. This means that the region of significant influence will be larger than the actual vortex core and spread out over regions separated by topology. This demonstrates that the way of thinking of engineers simulating and analyzing flow is often not topology based, which may lead to problems applying topology to answer their questions concerning the flow.

\subsection{Topology and Velocity}

In this subsection, the relation of the velocity magnitude of the flow to the resulting topological information is analyzed. For this, we compare two streamlines in two fields with different velocity magnitudes but the same velocity directions. Let $D \subset \mathbb{R}^{d}$ be an open domain and $v: D \rightarrow \mathbb{R}^{d}$ a vector field satisfying the Lipschitz condition. Let $Z:=\{z \in D \mid v(z)=0\}$ be the set of critical points and $D_{\varepsilon}:=\{x \in D|\forall z \in Z:| x-z \mid>\varepsilon\}$ the domain without the critical points and their $\varepsilon$-neighborhood. In the following, we compare the two vector fields

$$
\begin{aligned}
& \bar{v}: D_{\varepsilon} \rightarrow \mathbb{R}^{d}, x \mapsto v(x) \\
& \tilde{v}: D_{\varepsilon} \rightarrow \mathbb{R}^{d}, x \mapsto \frac{v(x)}{|v(x)|} .
\end{aligned}
$$

For $a \in D_{\varepsilon}$ and $\bar{I}=\left(0, t_{\max }\right)$ let $\bar{c}_{a}: \bar{I} \rightarrow D_{\varepsilon}$ be the well-defined streamline of $\bar{v}$ through $a$ with maximal length, that is

$$
\begin{aligned}
\bar{c}_{a}(0) & =a \\
\frac{\partial \bar{c}_{a}}{\partial t}(t) & =\bar{v}\left(\bar{c}_{a}(t)\right)=v\left(\bar{c}_{a}(t)\right) .
\end{aligned}
$$

Define the following mapping for reparametrization:

$$
l: \bar{I} \rightarrow l(\bar{I}), \quad l(t)=\int_{0}^{t}\left|\bar{v}\left(\bar{c}_{a}(\tau)\right)\right| d \tau .
$$

This function is strictly monotonic and therefore invertible. We define

$$
\tilde{I}=\left(l(0), l\left(t_{\max }\right)\right)=l(\bar{I})
$$




$$
\tilde{c}_{a}: \tilde{I} \rightarrow D_{\varepsilon}, s \mapsto \bar{c}_{a}\left(l^{-1}(s)\right)
$$

Then $\tilde{c}_{a}$, a reparametrization of $\bar{c}_{a}$, is the well-defined streamline of $\tilde{v}$ through starting point $a$ :

$$
\begin{aligned}
\tilde{c}_{a}(0) & =\tilde{c}_{a}(l(0))=\bar{c}_{a}(0)=a \\
\frac{\partial \tilde{c}_{a}}{\partial s}(s) & =\frac{\partial \bar{c}_{a}}{\partial s}\left(l^{-1}(s)\right) \\
& =\bar{v}\left(\bar{c}_{a}\left(l^{-1}(s)\right)\right) \frac{1}{\frac{\partial l(t)}{\partial t}} \\
& =\bar{v}\left(\bar{c}_{a}\left(l^{-1}(s)\right)\right) \frac{1}{\left|\bar{v}\left(\bar{c}_{a}\left(l^{-1}(s)\right)\right)\right|} \\
& =\bar{v}\left(\tilde{c}_{a}(s)\right) \frac{1}{\left|\bar{v}\left(\tilde{c}_{a}(s)\right)\right|} \\
& =\tilde{v}\left(\tilde{c}_{a}(s)\right) .
\end{aligned}
$$

This means that the streamlines of $\bar{v}$ are the same as the streamlines of $\tilde{v}$. Thus, the velocity magnitude of the whole field can be artificially set to one and, in contrast to vorticity and other quantities, streamlines and flow topology will stay the same. This reveals that flow topology is independent of the velocity magnitudes of a vector field and thus shows that topology is ill suited to describe or analyze features whose models strongly depend on the velocity magnitude of the flow.

\subsection{Determining Parameters}

The center of a pure Vatistas vortex is a critical point in $2 \mathrm{D}$, and thus the position of the vortex can be easily determined using vector field topology. In contrast to other feature definitions based on vorticity or pattern matching, the center position is automatically determined with subpixel accuracy. This is a distinct advantage of topology as subpixel accuracy is often hard to obtain [4]. However, topology is sensitive to noise, therefore subpixel results are meaningless for noisy data such as those obtained by measurements where the only solution is smoothing the data.

The determination of the size of a vortex - or its vortex core region is much more challenging. For a pure 2D Vatistas Model, no size can be determined using topological methods. There are closed orbits at all distances to the center and so separatrices in this case. When two vortices interact, often a saddle point confines the regions of the two vortices. The separatrices as defined by the saddle point seem to enclose the vortex regions. However, in the case of a spiraling separatrix, no size can be determined numerically.

Comparing these quantities with the Vatistas parameters again reveals the two different approaches taken by topology and feature based descriptions. Topology segments the vector field into regions of same flow behavior, that is 
every particle within the vortex region as defined by topological methods will pass into the critical point or has emerged there. The vortex core radius as defined by the Vatistas vortex can be either larger or smaller than that region, as the topology depends entirely on the streamlines while for the Vatistas vortex, only the projection of the vectors onto a perfect rotation is evaluated (Figure 2). This aspect is detailed further in Section 4.

From this discussion it can be seen quite clearly that topology and feature models from engineering take quite different perspectives on the definition of interesting features. The first globally describes regions of same flow behavior in relation to inflow and outflow regions while the other is more interested in local properties like velocity and vorticity, which can not be determined using topology based methods at all (Section 3.2). However, it is application dependent which of these two perspectives is more appropriate or beneficial.

\section{Superposition Effects}

In this section, we will discuss the two different perceptions of vector fields mentioned in the previous section: the usual point of view based on streamline behavior and the perception of complex vector fields as a superposition, or linear combination, of several, possibly simpler vector fields (see Figure 1). We will call the first interpretation interaction view and the latter superposition view. Furthermore, the resulting effects for analysis and visualization using topology or other feature based methods are shown.

The difference between the two views becomes clear by looking at the occurrence of two or more features in close vicinity. The superposition view emphasizes the original features and their parameters. The interaction, on the other hand, describes how these features influence each other, by overlapping, friction and other phenomena, resulting in the actually observed flow. Up to now, nearly always only the interaction view has been studied and visualized. But the user is not only interested in the actual flow, but in explanations and, possibly simplified, models of the underlying phenomena.

In 2001 an international cooperative research program called HART II was conducted to investigate the physics of blade pressure, noise radiation, and vibrations caused by the wake of helicopter rotors $[1,4,16]$. Three-component Particle Image Velocimetry (3-C PIV) was part of the measurements. In the resulting vector fields superposition phenomena are ubiquitous (Figure 3) due to the overall velocity of the flow in the wind tunnel. Furthermore, each crossing of a blade creates, among other things, a new vortex which is added to the flow created by previous blade crossings, the movement and the shape of the helicopter. To understand the wake of the rotor blades, and to be able to create a model of it, all vortices and other features have to be detected and their parameters have to be determined. For accurate determination of the parameters, the superposition effects and their consequences for the accuracy of the analysis methods have to be studied. 

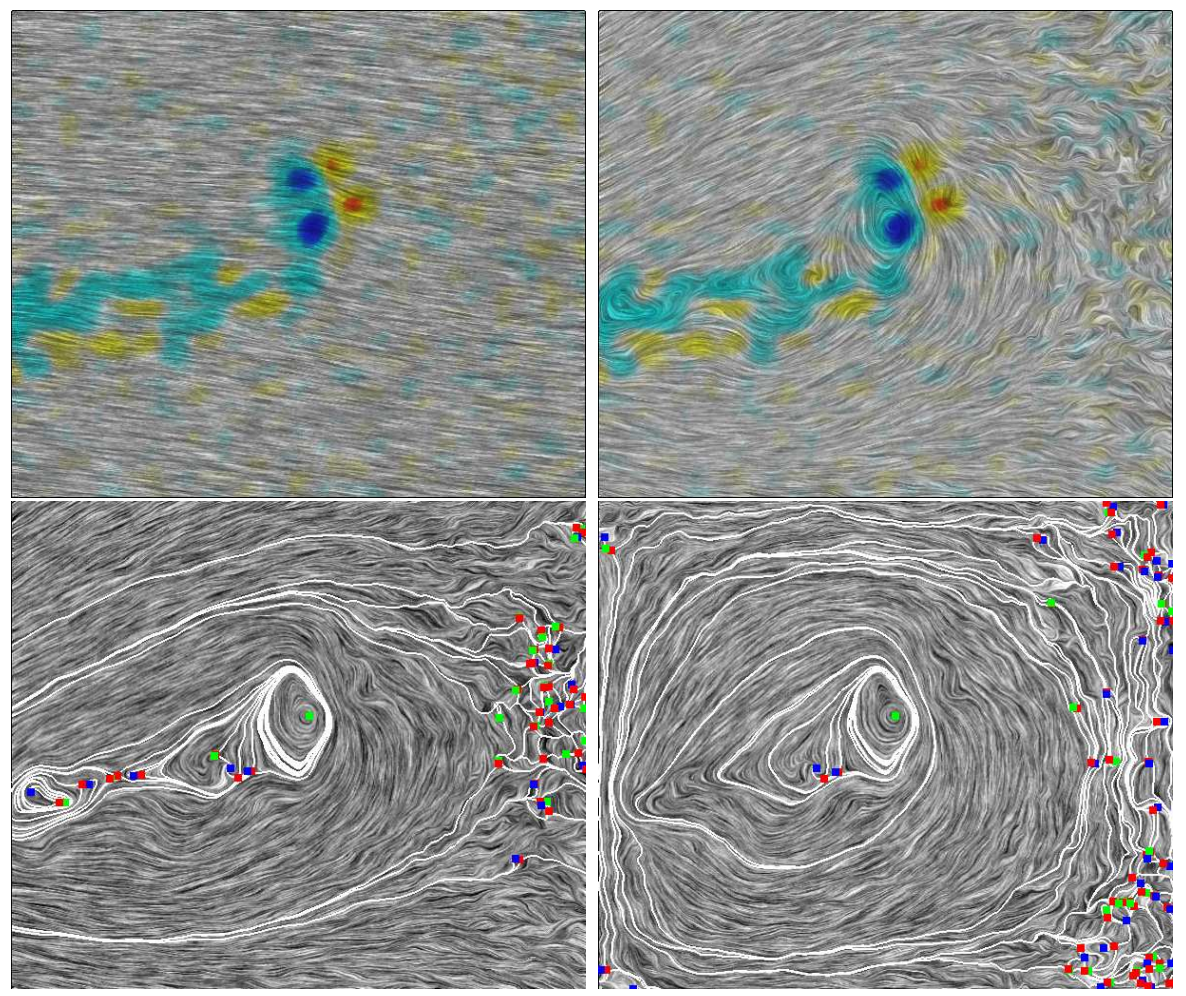

Fig. 3. One vector field of the HART II test measurements. Top left: LIC and vorticity of the original data set, dark blue: high negative vorticity, red: high positive vorticity. Top right: Vorticity and LIC of the data set after removing the average. Bottom left: LIC and topology after removing the average. Bottom right: LIC and topology of the region-specific flow of the dataset.

\subsection{Localized Flow Analysis}

Superposition is an effect most common in wind tunnel measurements. Dominant passing flow induced by the blower often hides vortices and other features so a direct visualization may not reveal all features or even none at all $[4,15]$. Regarding vortex detection this is most often dealt with by using vorticity or $\lambda_{2}$ as these quantities are Galilean invariant and thus do not vary with added or subtracted constant flow. Another method is to compute the average and remove it from the vector field. But the vortices, though having zero average, can add to the average of the whole field as they are assumed to be spread out infinitely and only a part of the vortex, not having zero average, might be within the data set. Thus, removing the average will change the results of a latter analysis of the data. Furthermore, different vortices may appear or disappear when subtracting or adding different constant flows (see [15] and Figure 3). 
One approach to solve this problem is to divide the flow field into three fields containing the divergence, rotation and harmonic parts using the Hodge decomposition theorem $[7,8,14]$. Features are then detected as extremal points of the divergence and rotational field. However, it is not quite clear how analysis and visualization methods are effected by this decomposition.

A better solution is to remove the boundary induced flow to get a regionspecific or localized flow as discussed in [18]. The flow through the boundary of the region-specific field is zero. Vorticity and divergence, and thus the local features of the original flow, are preserved in the region-specific flow and can be visualized using topology (Figures 3 and 4 ). The region-specific flow thus is independent of superposed constant and homogenous flows and represents a basis with non-changing topology for fields with different superposed constant or homogenous flows.

\subsection{Superposition of Nearby Features}

Superposition and interaction can also take place without added constant flow, two vortices for example can influence each other strong enough to result in warped visualizations or hiding of one of the vortices. In Figure 3, the effects of removing the average or computing the localized flow are shown for one data set of the HART II test. Neither of the two techniques results in a vector
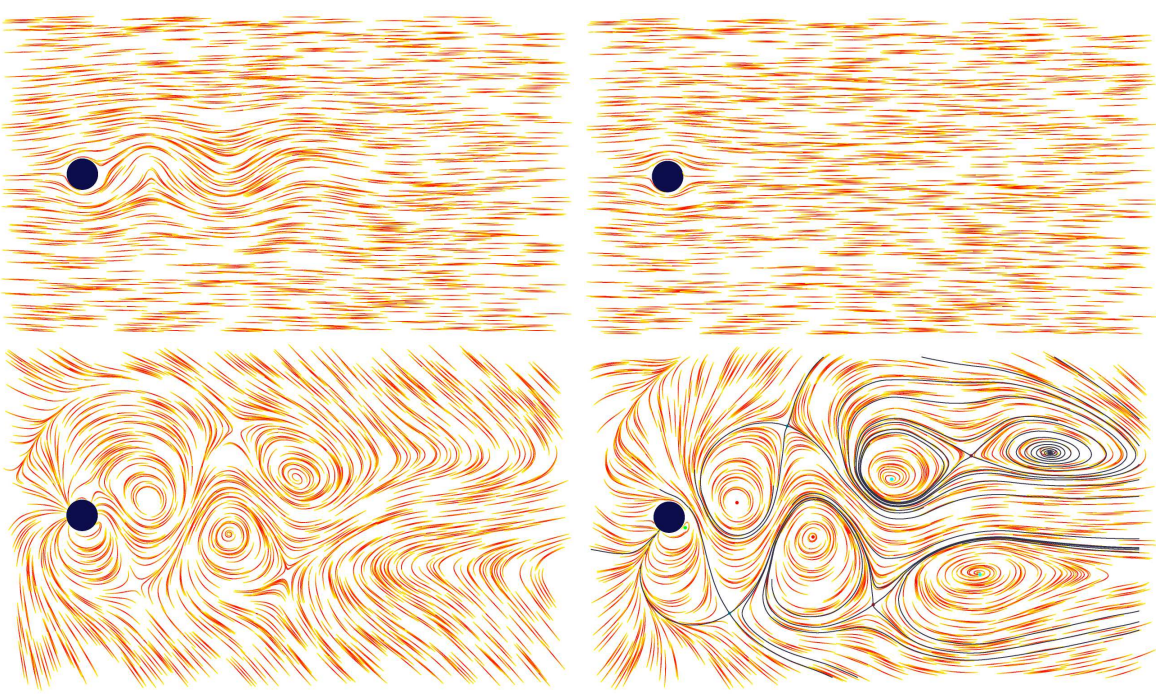

Fig. 4. Comparison of different fields obtained from a cylinder data set with a Kármán vortex street. Top left: Streamlines in the original flow. Only sinuous structures of the lines give hints on the vortices. Top right: Potential flow induced by the boundary. Bottom left: Three vortices revealed by removing the average flow. Bottom right: Subtracting the potential flow reveals all five vortices by use of topology. 
field where more than the most dominant vortex is found using topological methods. However, computing the vorticity, matching with a vortex mask, or evaluating the Vatistas vortex model directly reveals the multiple vortices the engineers examine in this data $[1,16]$. Therefore, local superposition effects caused the hiding of these vortices.

The authors generated some test data sets using different Vatistas vortices [17] to qualify and quantify the effects caused by superposition. The first two data sets consist of a weak vortex with radius $\mathrm{r}=2$ and radial velocity $v_{r}=1$ and a stronger vortex with $\mathrm{r}=5$ and $v_{r}=3$, both with $\mathrm{N}=1$. In the first data set (Figur 5, top left), both vortices have same rotation direction, and in the second data set (Figure 5, bottom left), the rotation direction differs. In both images, stramline based approaches like topology detect one vortex only. Template matching with a $3 \times 3$ rotation mask detects both vortices.

The accuracy of current feature definitions for the determination of the parameters of the underlying features was studied as well (Figure 5, right). Here, data sets consiting of two vortices with $\mathrm{r}=5, r_{c}=1$, and $\mathrm{N}=1$, were generated. Template matching with a $3 \times 3$ rotational mask detects the true vortex centers (Figure 5, top right). Setting all velocity magnitudes in the field to one, and matching afterwards, yields results more similar to the topological features (Figure 5, bottom right).

Topological and local streamline based features are good at detecting and describing features of the resulting flow, but not for features hidden or moved by superposition. The resulting errors in the analysis of the feature parameters are high in the case of superposition, even resulting in not detecting a vortex at all. Vorticity and template matching clearly depict the vortices in the resulting scalar fields, but even these results can be influenced by superposition. Assimilation effects, like two vortices with same parameters but different rotation direction which cancel each other out completely, effect the results of feature detection using any feature definition. Comparing the definitions for their robustness to these annihilation effects, template matching using vector valued masks gives the best results as it is tuned to the projection of the features to the abstract model as described in Section 3.1.

These effects should be considered when using topology or other methods for the visualization for vector fields. Again, though the superposition perception of vector fields can be appropriate for some applications, for others the actual streamlines are of higher importance. There, streamline based approaches for visualizing the flow, like topological methods, are better at describing the flow than velocity and direction based feature models like the Vatistas vortex.

\section{Conclusion}

In this paper, the authors have investigated the borders of hitherto existing topological methods on flow fields. Some of the aspects discussed here may ex- 


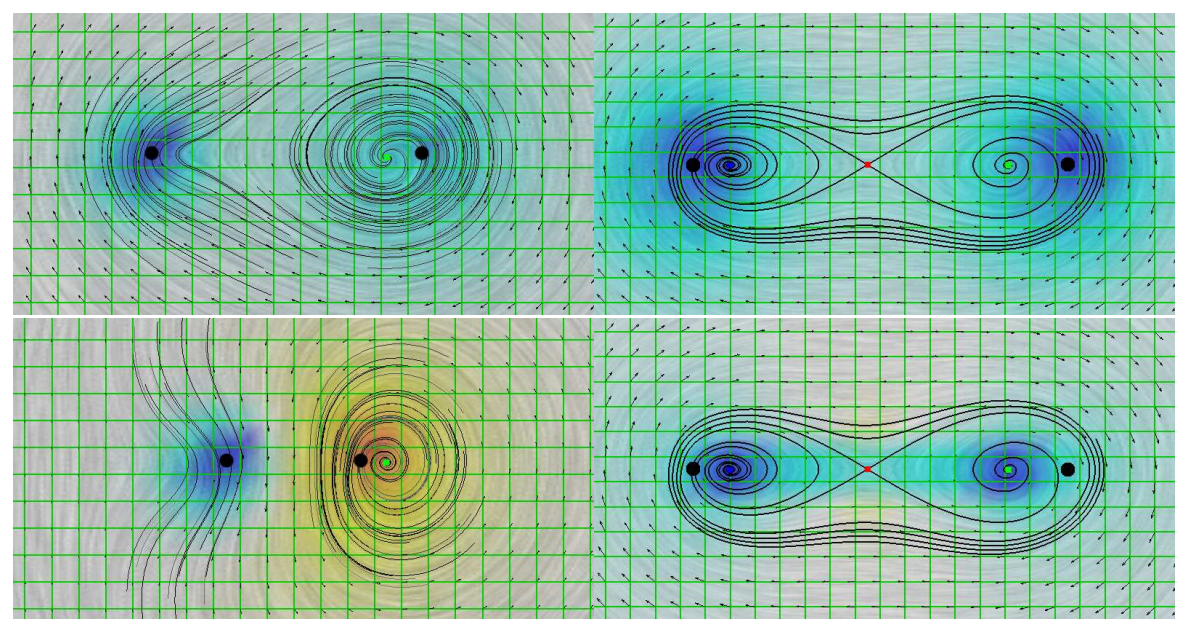

Fig. 5. Superposition and interaction of two Vatistas vortices [17]. All images: The original vortex centers are displayed as black dots. Grid (green), hedgehogs (black arrows), color coding of similarity to a $3 \times 3$ rotational mask from high negative values (blue) to high positive values (red). Left: The stronger vortex hides the weaker vortex in streamline based visualizations. Topology only detects one center each (green dot), therefore some streamlines are added. Top right: Template matching detects the true vortex centers. Bottom right: Setting all velocity magnitudes in the field to one, and matching afterwards, yields results more similar to the topological features.

plain why topology is not used more often by engineers. Engineers often think in terms of velocity, vorticity, and resulting feature models like the Vatistas vortex which are more tuned to a superposition perspective of the flow field. Parameters of the feature models thus can often not be determined or visualized using topological methods. Methods based on other feature definitions like pattern matching approaches have to be used then.

Nevertheless, topology is a useful tool for a first overview of and a detailed look at a data set when streamlines or the actual flow is to be studied. Furthermore, when analyzing or visualizing a data set, usually more than one method is used.

\section{Acknowledgments}

We thank Markus Rütten, DLR Göttingen, for the Kármán vortex street. Further thanks go to Dr. Berend van der Wall, DLR Braunschweig, and the whole HART II team for data of the HART II test. Furthermore, we like to thank the members of the FAnToM development team at the Universities of Kaiserslautern and Leipzig for their help with programming. 


\section{References}

1. C. L. Burley, T. F. Brooks, B. G. van der Wall, H. Richard, M. Raffel, P. Beaumier, Y. Delrieux, J. W. Lim, Y. H. Yu, C. Tung, K. Pengel, and E. Mercker. Rotor wake vortex definition - initial evaluation of results of the HART-II study. In 28th EUROPEAN ROTORCRAFT FORUM, Bristol, England, 2002.

2. J. Ebling and G. Scheuermann. Clifford convolution and pattern matching on vector fields. In Proceedings of IEEE Visualization 2003, pages 193-200, Los Alamitos, CA, 2003. IEEE Computer Society.

3. J. Ebling and G. Scheuermann. Fourier transform on multivector fields. IEEE Transactions on Visualization and Computer Graphics, 11(4):469-479, 2005.

4. J. Ebling, G. Scheuermann, and B.G. van der Wall. Analysis and visualization of 3-c piv images from HART II using image processing methods. In Data Visualization 2005, pages 161-168, 2005.

5. E. B. Heiberg, T. Ebbers, L. Wigström, and M. Karlsson. Three dimensional flow characterization using vector pattern matching. IEEE Transactions on Visualization and Computer Graphics, 9(3):313-319, 2003.

6. J. Jeong and F. Hussain. On the identification of a vortex. Journal of Fluid Mechanics, 285:69 - 94, 1995.

7. K. Polthier and E. Preuß. Variational approach to vector field decomposition. In Data Visualization 2000, pages 113-134, 2000.

8. K. Polthier and E. Preuß. Identifying vector field singularities using a discrete hodge decomposition. Visualization and Mathematics III, pages 775-792, 2003.

9. F. H. Post, B. Vrolijk, H. Hauser, R. S. Laramee, and H. Doleitsch. The state of the art in flow visualization: Feature extraction and tracking. Computer Graphics Forum, 22(4):775-792, 2003.

10. W.J.M. Rankine. Manual of Applied Mechanics. C. Griffen Co., London, 1858.

11. M. Roth. Automatic Extraction Of Vortex Core Lines And Other Line Type Features For Scientific Visualization. PhD thesis, ETH, 2000.

12. M.P. Scully. Computation of helicopter rotor wake geometry and it's influence on rotor harmonic airloads. Technical Report ASRL TR 178-1, Masachussetts Institute of Technology, March 1975.

13. B. Shneiderman. The eyes have it: A task by data type taxonomy for information visualizations. In In Proceedings of the IEEE Symposium on Visual Languages, pages 336-343, Washington, 1996. IEEE Computer Society Press.

14. Y. Tong, S. Lombeyda, A. Hirani, and M. Desbrun. Discrete multiscale vector field decomposition. ACM Transactions on Graphics, 22(3):445-452, 2003.

15. T. Urness, V. Interante, E. Logmire, I. Marusic, and B. Ganapathisubramani. Interactive poster: Illustrating different convection velocities of turbulent flow. In Poster Compendium of IEEE Visualization 2004, Los Alamitos, CA, 2004. IEEE Computer Society Press.

16. B. G. van der Wall, C. L. Burley, Y. H. Yu, H. Richard, K. Pengel, and P. Beaumier. The HART II test - measurement of helicopter rotor wakes. Aerospace Science and Technology, 8(4):273-284, 2004.

17. G.H. Vatistas. New model for intense self-similar vortices. Experiments in Fluids, 14(4):462-469, 1998.

18. A. Wiebel, C. Garth, and G. Scheuermann. Localized flow analysis of $2 \mathrm{~d}$ and 3d vector fields. In Data Visualization 2005, pages 143-150. Eurographics Association, 2005. 